\title{
A BANACH ALGEBRA GENERALIZATION OF CONFORMAL MAPPINGS OF THE DISC
}

\author{
BY \\ RICHARD ARENS
}

1. Introduction. In [II] there was introduced a very special type of Banach algebra analogous to the algebra of functions

$$
f(z)=\sum_{n=0}^{\infty} a_{n} z^{n}
$$

holomorphic for $|z|<1$ but continuous for $|z| \leqq 1$. The generalization proceeded by replacing the integers $n$ in 1.1 by elements from some distinguished sub-semi-group of some fixed abelian group $G$. (More details below in this section.)

The space $\Delta$ of maximal ideals of such a Banach algebra $A$ is the analogue of the closed unit disc $|z| \leqq 1$; and there is a well-known correspondence between conformal self-mappings of $\Delta$ and algebra-automorphisms of $A$.

This paper is devoted to discovering the form of these "generalized conformal" mappings of $\Delta$, or automorphisms of $A$. When the group of exponents (or of powers) is discrete and archimedean, there emerges a simple construction of all those that leave a certain set $\Omega$ (one analogue of the origin in the classical case) within itself.

Algebras AP of holomorphic functions uniformly almost periodic in a halfplane satisfy our conditions. Moreover, the set $\Omega$ is then merely one point; and (except in essentially the case 1.1) all automorphisms necessarily leave it fixed. Thus all automorphisms of AP are exhibited.

One particular algebra, based on a nonarchimedean, totally ordered group of exponents, is studied in detail in order to establish the natural limits for the results obtained for the archimedean ordered (in the large sense of "partially ordered") case.

The tools prepared and used are results on the form of "generalized holomorphic" functions which are of absolute value 1 on the boundary, and vanish only at 0 , etc. It would be unfair not to admit that our theorems here are based on the work of Harald Bohr and others on holomorphic almost periodic functions, as set forth in [III].

We exhibit a large class of bounded derivations in $A$ at points of $\Delta$. Naturally, there are none at points of the Silov boundary $\Gamma$ of $\Delta$. Except in the trivial case, there are none at the center of $\Delta$ for the AP algebras. This is why it is a fixed point.

Received by the editors October 25, 1955. 
Let us review briefly the essential definitions and notations of [II] that will be used here. Since we will be primarily interested in abelian discrete groups $G$, we will make our definitions here in that context.

There is supposed to be given in $G$ a semigroup $G_{+}$which generates $G$. The algebra $A_{1}$ is that subalgebra of $L_{1}(G)$ of functions which vanish outside $G_{+}$. The algebra $A_{1}$ can be represented (via the Fourier transform) as an algebra of continuous functions on the character group $\Gamma$ of $G$. The algebra $A_{0}$ is the uniform closure of that function algebra. The space of maximal ideals is the same for $A_{1}$ as for $A_{0}$, and is a compact set canonically homeomorphic to the set $\Delta$ of bounded complex valued homomorphisms of $G_{+} . \Delta$ contains $\Gamma$ in a natural way, since characters of $G$ are characters of $G_{+}$. Sometimes $G$ is (partially) ordered. Then, quite tacitly, $G_{+}$is to be taken as the elements greater than (or equal to) the identity.

We write all groups multiplicatively, and all neutral elements as 1 .

2. The connection with holomorphic almost periodic functions. Let $G$ be a totally, archimedean ordered abelian discrete group. Then $G$ is isomorphic to a subgroup $g$ of the additive reals (but we write $G$ multiplicatively). We may write this correspondence as follows. Let $\lambda$ belong to $g$. Then the corresponding element of $G$ will be denoted by $z^{\lambda}$.

According to the process exhibited in [II] and reviewed briefly above, we arrive at algebras $A_{1}, A_{0}$, of which $A_{0}$ can be regarded as an algebra of functions, sup-normed, on its own space of maximal ideals $\Delta$.

At this point, the reader should have firmly in mind what $A_{1}, A_{0}, \Delta, \Gamma$, etc. are when $G$ (or rather $g$ ) is the group of integers with the usual ordering.

The non-negative (real-valued) members $\rho$ of $\Delta$ are of special interest. In the present case there exists for each such $\rho$ a real number $b(0 \leqq b \leqq 1)$ such that, for $\lambda$ positive,

$$
\rho\left(z^{\lambda}\right)=b^{\lambda}
$$$$
\left(\lambda \in g, z^{\lambda} \in G_{+}\right) .
$$

Here $b^{0}=1$ even when $b=0$. When $0<b<1$, the $\rho$ gives (via 2.1) an isomorphism of $G_{+}$with a subgroup of the reals under multiplication.

We may suppose that $g$ contains the real number 1 , and select the $\rho$ for which the $b$ of 2.1 has the value $1 / e$. By [II, 7.4] there is a mapping

$$
M_{p}:[0, \infty] \rightarrow \Delta
$$

of the closed right half-plane into $\Delta$, in which

$$
M_{\rho}(w) \in \Delta, \quad M_{\rho}(w)(x)=\rho(x)^{w}
$$$$
\left(x \in G_{+}\right) .
$$

The mapping 2.2 can be extended to $\infty$, by setting $M(\infty)(x)=1$ or 0 according to whether $x$ is the identity in $G$ or not. This extended $M_{\rho}$ is continuous on $[0, \infty]$ if the topology is so defined that $w=u+i v$ approaches $\infty$ precisely when $u$ approaches infinity.

Any continuous function $\phi$ on $\Delta$ (say, a member of $A_{0}$ ) becomes upon sub- 
stitution of $M_{\rho}$ a holomorphic function on $(0, \infty)$, continuous on $[0, \infty]$ :

$$
N_{\rho}: \phi \rightarrow \phi \circ M_{\rho} \text {. }
$$

For example, let $\phi=\dot{x}$ (by which we mean that $\phi(\alpha)=\alpha(x)$, for some fixed $x$ in $G$ ). Suppose that $x=z^{\lambda}$ for some real number $\lambda$ in $g_{+}$, using the symbolic notation introduced earlier. It is to be verified directly that (for complex $w$ )

$$
N_{\rho}(\dot{x})(w)=e^{-\lambda w} .
$$

Thus, under the mapping $N_{\rho}$ (to be presently disclosed as an isomorphism), these generators pass into exponentials bounded on our half-plane. By well-known properties of almost periodic functions [III; p. 144], every member of $A_{0}$ passes over into a bounded member of u.a.p. [0, $[$ (to use the notation of [III; p. 142]). Moreover, if $\phi$ represents an element of $A_{1}$

$$
\phi=\sum a_{n} \dot{x}_{n}, \quad \sum\left|a_{n}\right|<\infty, \quad x_{n}=z^{\lambda_{n}}
$$

then the corresponding function is, by 2.3 ,

$$
\phi^{\prime}(w)=\sum a_{n} e^{-\lambda_{n} w}
$$$$
\left(-\lambda_{n} \in g_{+}\right)
$$

From this it can be seen that the "exponents" $\left\{-\lambda_{n}\right\}$ of 2.41 are negative (large sense!) numbers from the real module $g$; and the "Fourier coefficient" (in the sense of a.p. functions) of 2.41 corresponding to $-\lambda$ is the same as the Fourier coefficient of 2.4 corresponding to the element $x=z^{-\lambda}$. Conversely, given an element of u.a.p. $[0, \infty\rangle$ like 2.41 , with

$$
\sum\left|a_{n}\right|<\infty
$$

there is a $\phi$ as in 2.4 that gives rise to it. This proves the following:

2.5. THEOREM. The algebra $A_{1}$ is isomorphic and isometric with the algebra $A P_{1}(g)$ of u.a.p. $[0, \infty\rangle$ functions with absolutely convergent Dirichlet series (normed by the sum 2.42) whose exponents lie in the module $g$ and are negative.

The fact that the chosen $\rho$ assumes no value twice makes sure that $M_{\rho}$ maps the imaginary axis onto a dense subgroup of $\Gamma$, by [II; 7.3]. Hence for any continuous function $\phi$, the least upper bound of $\left|N_{\rho} \phi(i v)\right|$, for all real $v$, is the same as the maximum of $\phi(\alpha), \alpha \in \Gamma$. Thus 2.2 is norm-preserving from $A_{0}$ to the ensuing algebra of almost periodic functions. The complete statement is the following:

2.6. ThEOREM. The algebra $A_{0}$ is isomorphic and isometric with the algebra $A P_{0}(g)$ of u.a.p. $[0, \infty)$ functions whose exponents lie in the module $g$ and are negative. The norm in the latter algebra is the least upper bound of the absolute values.

The "onto" part of 2.6 follows from the fact that $N_{\rho}\left(A_{1}\right)$ is already dense in $\mathrm{AP}_{0}(g)$. 
Of course $\mathrm{AP}_{1}(g)$ is a subalgebra of $\mathrm{AP}_{0}(g)$, just as $A_{1}$ is contained in $A_{0}$. It is also to be borne in mind that the members of $\mathrm{AP}_{0}(g)$ are holomorphic on the half-plane $[0, \infty)$.

Points of $[0, \infty]$ evidently determine points of the structure space $\Delta$ of the $\operatorname{AP}_{i}(g)(i=0,1)$, but they fill merely a dense set. It is therefore of interest to note that an element of $\mathrm{AP}_{i}(g)$ which has no inverse in that algebra has to vanish not merely somewhere on $\Delta$ but actually somewhere on $[0, \infty]$ (see [III, pp. 144-145]). We continue this line of reasoning as follows.

2.7. THEOREM. Let $f$ belong to $A P_{i}(g)(i=0$ or 1$)$ and suppose it never vanishes on $[0, \infty]$. Then there is an $h$ in $A P_{i}(g)(i=0$ or 1 , respectively) such that $f=e^{h}$.

The proof we have in mind is rather round-about. We first observe that this $f$ has an inverse in its algebra. Next we observe the structure space $\Delta$ is simply connected, by virtue of the polar decomposition [II; 3.2]. Hence invertible elements have logarithms $[\mathrm{I} ; 8.1]$. Our real object is the following.

2.8. Lemma. Let $f$ be a bounded u.a.p. $[0, \infty)$ function with

$$
\begin{aligned}
& |f(i v)|=1, \\
& |f(u+i v)| \neq 0 \\
& \text { for all } u \geqq 0 \text {. }
\end{aligned}
$$

Then $f$ is of the form

$$
f(w)=c e^{\lambda w} \quad(\lambda \text { real, }|c|=1) .
$$

Proof. By [III, p. 159] all the exponents are negative. By 2.82 and [III, p. 162] there is among the exponents actually occurring a numerically least one, $\lambda$. Then

$$
f(w)=f_{1}(w) e^{\lambda w}
$$

where

$$
f_{1}(w) \sim c+\sum_{\mu<0} c_{\mu} e^{\mu w} \quad(c \neq 0) .
$$

Hence $f_{1}(w)$ never vanishes even on $[0, \infty]$. By 2.7 , it is of the form $e^{h}$, $h \in$ u.a.p. $[0, \infty\rangle$ and also bounded. By $2.81, i h(i v)$ is real, so that its nonzero Fourier coefficients depend hermitean-symmetrically on the exponents. On the other hand, by [III, p. 159] the exponents are negative. Thus $h$ is constant, and so is $f_{1}$. This substantially completes the proof.

This result may be reformulated by saying that if $f$ is a representative function of $A_{0}$ whose "boundary" values are of absolute value 1 , which has no zeros except at the "center" $\rho_{0}$ of the "disc" $\Delta$, and if $f$ has the value 1 at the point 1 in $\Gamma$, then there is an $x$ in $G$ such that $f(\zeta)=\zeta(x)$ for all $\zeta$ in $\Delta$. We postpone the formal statement until the next section where we shall treat a more general type of $G, G_{+}$combination. 
3. Characterization of powers in case of partially ordered $G$. Let $S$ be any set, and let $T$ be the additive group of real-valued functions on $S . T$ can be (partially) ordered in an obvious way. Let $U$ be any semi-group of positive clements (i.e., $\geqq 0$ ). This $U$ generates a subgroup $V$ of $T$ such that

3.11. Every element is a difference of positive elements;

3.12. Given $v \neq 0$ there is a homomorphism $h$ into the additive reals for which $h(v) \neq 0$ and $h(t) \geqq 0$ for $t \geqq 0$.

For 3.12, just select a point $s$ of $S$ where $v(s) \neq 0$ and let $h(t)=t(s)$.

Conversely, any ordered abelian group $V$ satisfying $3.11,3.12$, cf. [II; 8.4 ], can be obtained in this way. We shall call such a group weakly archimedean ordered, because there are sufficiently many homomorphisms into archimedean groups. If the group is written multiplicatively, $3.11,3.12$ must be restated in a simple way. If $G$ is an abelian weakly archimedean ordered discrete group, and $G_{+}$is the class of positive elements, then the functionals demanded in 3.12 permit the formation of many elements $\rho$ of the corresponding $\Delta$,

$$
\rho(x)=e^{-h(x)} .
$$

In the general context of [II], the points $\zeta$ of $\Delta$ may be classified according to whether $\zeta(x)$ never vanishes for $x$ in $G_{+}$(for example, the elements of $\Gamma$ or those given by 3.2 in the context of this section) or whether it sometimes vanishes. If $\zeta(x)=0$ for some $x$ in $G_{+}$we shall call $\zeta$ singular. The totality of singular characters of $G_{+}$we shall denote by

$\Omega$.

Of course $\Omega$ is a subset of $\Delta$. In fact, in the natural multiplication of $\Delta, \Omega$ is an ideal: $\Omega \Delta \subset \Omega$. We use $\Omega$ to remind us that the "origin" (or "center") $\rho_{0}$ belongs to $\Omega$. Naturally $\rho(x)$ does not need to vanish for all $x$ when $\rho \in \Omega$. Thus $\zeta(1)=1$ for all $\zeta$ in $\Delta . \Omega$ is the totality of zeros of all the powers $\dot{x}\left(x \in G_{+}\right)$.

In the case of 2 complex variables, when $\Delta$ is naturally embedded as a bicylinder, $\Omega$ is the class of points at least one of whose coordinates is 0 .

The nonsingular $\zeta$ can be immediately regarded as homomorphisms of all of $G$ into the nonzero complex numbers (bounded on $G_{+}$).

We shall need the following, which is an appropriate condensation of arguments used in [II, 5.6, 7.2, 8.4] and constitutes a strengthening of [II, 8.4].

3.3. Тниокем. Let $G$ be a weakly archimedean ordered discrete abelian group. Let $P$ be the class of positive nonsingular elements of $\Delta$. For each such $\rho$ let $H_{\rho}$ be the class of all $\rho^{i v}(-\infty<v<\infty)$, contained in $\Delta$. Then

3.31. $H_{\rho}$ is a subgroup of $\Gamma$; and

3.32. If any $\rho \in P$ is selected, then the union of all the $I_{\rho \sigma}(\sigma \in P)$ is a dense subgroup of $\mathrm{T}$.

Proof. $\rho^{i v}(x)=e^{i v \log x}$ clearly defines an element of $\mathrm{I}$, since $\rho(x)$ will not 
vanish. Considering now 3.32, we first observe that the union $H$ there mentioned is a subgroup of $\Gamma$. Indeed, if $(\rho \sigma)^{i u}$ and $(\rho \tau)^{i v}$ are multiplied, where $u \neq 0$, then the product is $\left(\rho \sigma(\rho \tau)^{w}\right)^{u i}$ where $w=v u^{-1}$, and thus lies in some $H_{\rho v}$.

Next, let $H$ be the subgroup formed by all these $H_{\rho \sigma}$. If $H$ is not dense in $\Gamma$, then by the duality theorem for the pair $\Gamma, G$ there is an element $x$ of $G$ such that $x(\alpha)=1$ for all $\alpha$ in $H$, while $x \neq 1$. Now construct an $h$ as given by 3.12 , such that $h(x) \neq 0$. We form $\sigma(y)=e^{-h(y)}$ (for all $y$ ), and we can surely find an $n=1$ or 2 such that

$$
\left(\rho \sigma^{n}\right)(x)=\rho(x) \sigma(x)^{n} \neq 1 .
$$

Therefore $\left(\rho \sigma^{n}\right)^{i v}(x) \neq 1$ for some real $v$. But this contradicts the statement that $x$ has always the value 1 on $H$. We conclude that $H$ is dense, as was to be shown.

In the application, the fixed $\rho$ involved here is of no interest, and can be taken as 1 . But it is of general interest that $\Gamma$ is a limit of the $H_{\rho}$ in the sense of decreasing $\rho$.

We can now formulate a characterization of those representative functions which have only one term in their expansion.

3.4. Theorem. Let $G$ be a weakly archimedean ordered discrete abelian group. Let $\phi$ be a representative function of an element of $A_{0}$. Then a necessary and sufficient condition that $\phi$ be of the form $c \dot{x}$ (i.e., $\phi(\zeta)=c \zeta(x)$ for all $\zeta$ in $\Delta$ ) is that

3.41. $|\phi(\alpha)|$ is constant on $\Gamma$,

3.42. $\phi(\zeta)$ never vanishes for nonsingular $\zeta$.

Proof. Let $\phi(1)=c$. If $c=0$ then $\phi$ vanishes on $\Gamma$ and hence is 0 . If $c \neq 0$, divide it out so that we may assume now

$$
\phi(1)=1 .
$$

Let $\rho, \sigma$ be selected from $P$ (see 3.3). Then, for complex numbers $z=x+i y$, $w=u+i v(x, u \geqq 0)$, the element $\rho^{z} \sigma^{w}$ of $\Delta$ is nonsingular, so that the continuous function

$$
f(z, w)=\phi\left(\rho^{z} \sigma^{w}\right)
$$

is holomorphic in each variable when $x, u>0$, never 0 , and has absolute value 1 when $x=u=0$.

When a fixed value of $w$ is selected, it is u.a.p. $[0, \infty\rangle$ relative to $z$ essentially, because $\Gamma$ is compact. If the value of $w$ is fixed at $i v$, then $f(, i v)$ satisfies the conditions of 2.8. Accordingly we have

$$
f(z, i v)=a(v) e^{\lambda(v) z}
$$

where $a(0)=1$ since it equals $\phi(1)$, and $\lambda(v)$ is always real and positive. Similarly, 


$$
f(i y, w)=b(y) e^{-\mu(v) w} .
$$

Equating these for $z=i y, w=i v$, we presently discover that $\lambda(v)=\lambda_{0}+c v$, $\mu(y)=\mu_{0}+c y$. Since $\lambda(v)$ is never negative, it must be that $\lambda(v)$ is constant. But we also find that

$$
a(v)=e^{-i \mu_{0} v} .
$$

Therefore, 3.45 takes th? form

$$
e^{-i \mu_{0} v-\lambda_{0} z}
$$

Since $f(z, w)$ is holomorphic in $w$, its value must be

$$
f(z, w)=e^{-\lambda_{0} z-\mu_{0} w} .
$$

From this we obtain at once the relation

$$
\phi\left(\rho^{z} \sigma^{w}\right)=\phi\left(\rho^{z}\right) \phi\left(\sigma^{w}\right) .
$$

Now let $\alpha, \beta$ be elements of the topologic group $\Gamma$. We can find a $\rho$ in $P$ and a real $u$ such that $\rho^{i u}$ is near $\alpha$ (by 3.3). Similarly, let $\sigma^{i v}$ be near $\beta$. Then $\rho^{i u} \sigma^{i v}$ is near $\alpha \beta$. It follows that

$$
\phi(\alpha \beta)-\phi(\alpha) \phi(\beta)
$$

is nearly 0 . Being constant, it is 0 . Hence $\phi$ is a continuous character of $\Gamma$. Thus there is an $x$ in $G$ (the character group of $\Gamma$ ) such that $\phi(\alpha)=\alpha(x)$ for all $\alpha$ in $\Gamma$. This is adequate to establish 3.4.

It will be shown later (5.8) that 3.4 with the "weak-archimedean" omitted, is not true; even when $G$ is required to be linearly ordered.

4. Automorphisms of $A_{0}, A_{i}$. A Banach algebra automorphism $U$ of $A$ induces a homeomorphism $U^{T}$ of $\Delta$, and thus induces an automorphism of $A_{0}$. Therefore, if we are going to expose the structure of automorphisms, it suffices to consider automorphisms of $A_{0}$. The corresponding homeomorphism $U^{T}$ is defined by

4.1

$$
U^{T}(\zeta)(\phi)=\zeta(U(f))
$$

where $f \in A_{0}$ and $\phi$ represents $f$ on $\Delta$. It is the analogue of a conformal mapping of the disc on itself. In general, $U^{T}$ sends boundary $(\Gamma)$ points into boundary points. Sometimes it is transitive on the remaining set, but not always (see §5).

We do not intend to classify all such "conformal mappings," but only those which correspond in the classical case to

\section{2}

$$
U^{T}(\zeta)=c \zeta
$$$$
(|c|=1) \text {. }
$$

The analogue of 4.2 in the general case is obtained as follows. Select an automorphism $a$ of the group $G$ which preserves $G_{+}$

$$
a\left(G_{+}\right)=G_{+} .
$$


Select also a fixed character $\alpha \in \Gamma$. Then let

$$
U(x)=\alpha(x) a(x) .
$$

This is an element of $A_{0}, A_{1}$ when $G$ is discrete. More generally, one has to define $U(f)$ for $f \in A_{1}$,

$$
U(f)=\int_{G_{+}} f\left(a^{-1}(x)\right) \alpha\left(a^{-1}(x)\right) d x .
$$

It is easily seen that 4.33 defines a continuous automorphism in $A_{0}$, and in $A_{1}$. Even when $G$ is not discrete, it is not misleading to look at 4.32 .

We shall discover the corresponding 4.1, evaluated at $x \in G_{+}$:

$$
U^{T}(\zeta)(x)=\zeta(U(x))=\alpha(x)(\zeta a(x))
$$

or, more briefly (to show the relation to 4.2 ),

$$
U^{T}()=\alpha \zeta \circ a .
$$

Here $\zeta \rightarrow \zeta \circ a$ is the dual (in $\Gamma$ ) of the automorphism in $G$. In the classical case there occurs none but the identity since

$$
\zeta \rightarrow \bar{\zeta}
$$

sends positive elements of $G$ (here, the integers) into nonpositive elements, violating 4.31 . Whether the $\alpha$ is absent in $4.32-4.35$ is readily checked, it depends only on whether

$$
U^{T}(1)=1
$$

If $a=1$, and $\Gamma$ is compact, we can think of $U^{r}$ as a rotation.

4.4. THEOREM. If $U$ is of the type 4.32-4.35, then $U^{T}$ sends the set $\Omega$ of singular elements of $\Delta$ into itself.

The proof can be omitted. Its converse is by no means trivial, and, in fact, false (see 5.7). However, the converse holds for groups of the type introduced in the preceding section.

4.5. THEOREM. Let $G$ be a weakly archimedean ordered discrete abelian group. Let $U$ be an endomorphism of the algebra $A_{0}$. Suppose the corresponding mapping $U^{T}$ (4.1) of the disc $\Delta$ leaves the singular element set $\Omega$ (3.21) in $\Omega$. Then $U$ is of the special form 4.32-4.33, and $U^{T}$ is of the form 4.34-4.35.

Proof. Let $x$ belong to $G_{+}$, and let $\phi$ be the function representing $U(x)$ on $\Delta$. Then $|\phi(\alpha)|=1$ on $\Gamma$ and $\phi(\zeta)$ never vanishes for nonsingular $\zeta$. By 3.4, $\phi(\alpha)=c \alpha(y)$ for every $\alpha$ in $\Gamma$, where $c$ (which depends on $x$ ) has absolute value 1 , and $y \in G_{+}$also depends on $x$. Writing $c$ as $\beta(x)$ and $y$ as $a(x), U(x)$ takes the form

$$
U(x)=\beta(x) a(x) .
$$


Considering that $U$ is a homomorphism, it appears that $\beta$ is a homomorphism of $G$ into the complex numbers (that is, $\beta \in \Gamma$ ) and $a$ is a homomorphism of $G$ into $G$ with 4.31 . If, moreover, $U$ is an automorphism, then $a$ is also an automorphism.

We now turn to a consideration of derivations in the algebras $A_{0}, A_{1}$. (The purpose is to show eventually that when $G$ is totally archimedean ordered, and has no least strictly positive element, then the sole singular element of $\Delta$ is necessarily fixed under any continuous automorphism.)

By a derivation at a point $\zeta$ in the structure space of an algebra $A$, we mean a linear functional $D$ satisfying

$$
D(f g)=D(f) g(\zeta)+D(g) f(\zeta) .
$$

Select any $\zeta$ from $\Delta$. Let its polar decomposition be $\zeta=\rho \alpha[$ II, 3.2]. Then a derivation $D_{\zeta}$ at $\zeta$ may be defined by

$$
D_{5}(\phi)=\frac{1}{2 \pi i} \int_{|w|=1} w^{-2} \phi\left(\alpha \rho^{1+w}\right) d w
$$

or, equivalently,

$$
=\left.D_{w} \phi\left(\alpha \rho^{1+w}\right)\right|_{w=0}
$$

for $\phi \in A_{0}$. The idea is of course that 2.22 sends $A_{0}$ into a class of holomorphic functions, and these may be differentiated. The derivation can then be drawn back into $A_{0}$, giving $\left({ }^{*}\right)$.

4.51. Lemma. $D_{5}$ is a bounded derivation in $A_{0}$.

This is clear.

4.52. Lemma. If $x \in G_{+}$and $|\zeta(x)| \neq 0,1$, then $D_{\zeta}(\dot{x}) \neq 0$.

Proof. On this hypothesis, $x$ passes into a nonconstant exponential function, whose derivative is not 0 .

A derivation is trivial if $D(\phi)=0$ for all $\phi$.

4.53. Lemma. If $|\phi(\zeta)|=\|\phi\|$ for some $\phi$ in $A_{0}$ and $\zeta$ in $\Delta$, then $D(\phi)=0$ in every bounded derivation of $A_{0}$.

Proof. First, $D\left(\phi^{n}\right)=n \phi(\zeta)^{n-1} D(\phi)$. We may suppose $\|\phi\|=1$. Then the right side is unbounded unless $D(\phi)=0$, while the left side is bounded.

The main consequence now comes.

4.54. Theorem. There are no bounded derivations of $A_{0}$ on the boundary $\Gamma$.

Proof. For each generator $\dot{x}$ of $A_{0}\left(x \in G_{+}\right)$, and each $\alpha \in \Gamma$, we have $|\dot{x}(\alpha)|=|\alpha(x)|=1=\|\dot{x}\|$, so that $D(x)=0$ for all of these. Hence $D=0$.

4.6. THEOREM. When $G$ is totally archimedean ordered, and there is no least 
strictly positive element, then there is no nontrivial bounded derivation at the center $\rho_{0}$ of $\Delta$.

Proof. At the "center" $\rho_{0}$, we have $\rho_{0}(x)=0$ for every $x \in G_{+}$except $x=1$. On our hypothesis, if $1<x$, then there are $y, z>1$ such that $x=y z$. Now, for a derivation at $\rho_{0}$

$$
D(x)=\rho_{0}(y) D(z)+\rho_{0}(z) D(y)=0 .
$$

By continuity, $D\left(A_{0}\right)=0$. [We thank I. M. Singer for this theorem.]

4.7. TheOREM. When $G$ is totally archimedean ordered with no least strictly positive element, and $U$ is an automorphism of $A_{0}$, then $U^{r}$ leaves the (unique) singular element $\rho_{0}$ of $\Delta$ fixed.

Proof. $U^{T}$ sends $\Gamma$ onto $\Gamma$. Wherever $U^{T}\left(p_{0}\right)$ is, there cannot be any bounded derivation, since there is none at $\rho_{0}$. But for $\zeta \neq \rho_{0}, \zeta \in \Gamma$, the formula 4.5 provides, according to 4.52 , a bounded nontrivial derivation. Hence $U^{T}\left(p_{0}\right)$ must be $\rho_{0}$ itself.

We now propose to see what these results mean for the algebras $\operatorname{AP}_{0}(g)$, $\operatorname{AP}_{1}(g)$ defined in $2.5,2.6$. We hereby expressly exclude the case in which these functions are all periodic. Then the module $g$ has no first strictly positive element. Hence 4.7 and 4.5 tell us what the automorphisms are. First let us recognize what $G$ is. We might as well take it to be the exponential functions

$e_{\lambda}$

$(\lambda \in g)$

where $e_{\lambda}(w)=e^{-i \lambda w}(w=u+i v)$. Given an automorphism $U$ of $\mathrm{AP}_{0}(g)$, let us look at 4.32. Here we see $\alpha$, a character, and $a$, an automorphism, of $G$. We may, however, regard them as defined and with values in $g$. The structure of $a$ is made clear by 4.31 : there must be a real positive number $m$ such that

$$
m g \subset g, \quad m^{-1} g \subset g \text { and } a(\lambda)=m \lambda .
$$

As to $\alpha$, nothing more can be said except that

$$
\alpha(\lambda+\mu)=\alpha(\lambda) \alpha(\mu), \quad|\alpha(\lambda)|=1 \quad(\lambda, \mu \in g) .
$$

Thus the general form of an automorphism of $A P_{0}(g)$, or of $A P_{1}(g)$, is (see 4.8, $4.81,4.82$ )

$$
\left.U\left(e_{\lambda}\right)=\alpha(\lambda) e_{m \lambda} \quad m \text { (fixed, }>0\right) .
$$

If it should happen that $\alpha$ has the form $\alpha(\lambda)=e^{i \lambda k}$ (any real $k$ might occur), then $U\left(e_{\lambda}\right)$ is simply the function whose value at $w$ is

$$
e^{i \lambda(m v+k)} \text {. }
$$

In this case, $U$ could be said to be induced by dilation and translation in the (imaginary) axis, but this case is not the general one (see 4.82). However, 
this type of automorphism permutes the maximal ideals corresponding to the places on the right half-plane among themselves. The linear transformation of that half-plane is easily discovered on inspecting 4.84 .

The most interesting observation is that there are automorphisms of $\mathrm{AP}_{0}(\mathrm{~g})$ which do not correspond to transformations of the half-plane.

The points of $\Delta$ form sets of imprimitivity, under the various $U^{T}$. The origin is one such set. For any $\zeta$, the set of imprimitivity to which it belongs is the class of all $\zeta \rho^{w}$ where $\rho$ is a positive real member of $\Delta$ not belonging to $\Gamma$, and $w$ is allowed.to vary over the half-plane. The origin, $\Gamma$, and the remainder form sets of transitivity.

5. A nonarchimedean example. Let $\Gamma$ be the set of pairs $(z, w)$ of complex numbers such that $|z|=|w|=1$. Let $f, g$ be the functions on $\Gamma$ defined by

$$
f(z, w)=z, \quad g(z, w)=w .
$$

Order the group $G$ of pairs of integers $(m, n)$ by saying that

$$
(m, n) \geqq(0,0) \text { if } m>0 \text { or } m=0 \text { and } n>0 \text {. }
$$

Let $A_{0}$ be the algebra obtained by closing under the uniform norm the linear combinations of the functions

$$
f^{m} g^{n} \quad \text { where }(m, n) \geqq(0,0) .
$$

This is evidently the $A_{0}$ constructed in the sense of this paper, based on $G$ with the total ordering 5.11 (except that $G$ is written additively. One could therefore replace $G$ by the functions 5.12.)

5.2. Theorem. The mapping wherein $\zeta$ is sent into $(\zeta(f), \zeta(g))$ is a homeomorphism of $\Delta$ with the set of pairs $(a, b)$ of complex numbers for which $|a|$, $|b| \leqq 1$ and also

$$
a=0 \text { if }|b|<1 \text {. }
$$

Proof. The mapping is obviously continuous. We therefore proceed to show that it is one-to-one and exhausts the set mentioned.

Consider the equation (for $n \geqq 0$ )

$$
\zeta\left(f g^{-n}\right) b^{n}=\zeta\left(f g^{-n} g^{n}\right)=a .
$$

If $|b|<1$ then (the first factor being bounded) $a=0$. Suppose next that $\zeta(f)=\eta(f)=a, \zeta(g)=\eta(g)=b$. Then $\zeta, \eta$ agree on elements 5.12 where $n \geqq 0$. But suppose

$$
5.22 \quad \zeta\left(f^{m} g^{-n}\right) \neq \eta\left(f^{m} g^{-n}\right) .
$$

Multiplying by $b^{n}$ on both sides gives

$$
\zeta\left(f^{m}\right) \neq \eta\left(f^{m}\right)
$$

unless $b=0$. Since 5.23 is impossible we get $b=0$ and (by 5.21) $a=0$. But then 


$$
\zeta\left(f^{m} g^{-n}\right) b^{n}=\zeta\left(f^{m}\right)=0
$$

and the same for $n$, denying 5.22 . Hence each pair $(a, b)$ comes from just one $\zeta$, and this can be defined for $(m, n) \geqq(0,0)$ by

$$
\zeta\left(f^{m} g^{n}\right)=0 \text { if } a=0, \quad=a^{m} b^{n} \text { if } a \neq 0 .
$$

5.3. Corollary. The part of $\Delta$ with $|a|>0$ is 3-dimensional, and that part with $|b|<1$ is 2-dimensional.

The proof rests on the fact that $|b|<1$ implies $a=0$.

5.31. Lemma. Terms like $f^{m} \phi(g)$ can occur in $A_{0}(m>0)$, where $\phi$ is any continuous function defined on the unit circle (not the disc!), and

$$
\zeta\left(f^{m} \phi(g)\right)=a^{m} \phi(b) \text {, or } 0 \text { if } a=0 .
$$

This is clear because $\phi$ is a limit of polynomials in $g$ and $g^{-1}$.

Now let $U$ be an automorphism of $A_{0}$. In fact, let

$$
\begin{aligned}
& U(f) \sim F(f, g), \\
& U(g)=H(f, g),
\end{aligned}
$$

where $F, H$ are Fourier series containing only terms of the type 5.12.

Let us consider where $U^{T}$ sends the point of $\Delta$ with coordinates $(a, b)$. Formally, by 5.32 ,

$$
U^{T}(\zeta)=(\zeta(U(f)), \zeta(U(g)))=(F(a, b), H(a, b)) .
$$

We can use these remembering they are only Fourier series. The set $a \neq 0$, being 3-dimensional, has to go into itself, which is to say that for every $b$, if $a \neq 0$ then the power series (in $a) F(a, b)$ cannot vanish. Hence $F(a, b)$ $=F(b) a^{m}$ where $m \geqq 0$ and $F$ is continuous on $|b|=1$. When $|a|=1$ and $|b|=1$ this $|F(a, b)|$ has also to be 1 , since the boundary $\Gamma$ goes into itself. Hence $|F(b)|=1$. (There is no need to worry about defining $F(b)$ for $|b|<1$, for then $a=0$.)

Leave $F$ for a moment, and consider that if $a=0$ then $H(a, b)$ needs absolute value 1 . Being without negative powers of $a$, it must depend merely on $b$ : call it $H(b)$. Now consider the part $\Omega$ where $a=0$. This is a unit disc, and $H(b)$ must be holomorphic, $1-1$, and "onto" there. Hence $H(a, b)$ reduces to

$$
H(b)=c\left(b-b_{0}\right)\left(1-b_{0} b\right)^{-1} \quad(|c|=1) .
$$

The whole mapping, as matters now stand, sends $(a, b)$ into

$$
\left(a^{m} F(b), H(b)\right)
$$$$
(m \geqq 0) .
$$

One-to-one-ness requires $m=1$. We summarize the result.

5.4. Theorem. Let $U$ be an automorphism of $A_{0}$. Then 


$$
U(f)=f F(g)
$$

and

$$
U(g)=c\left(g-b_{0}\right)\left(1-b_{0} g\right)^{-1}
$$

where $F$ is a continuous complex valued function defined on the unit circle, with $|F|=1,|c|=1$, and $\left|b_{0}\right|<1$. Moreover, 5.41 always defines an automorphism.

Proof. Only the last has not been considered. But the expressions $U(f)$, $U(\mathrm{~g})$ given in $\mathbf{5 . 4 1}$ are elements of $A_{0}$, and so $U$ is at least an endomorphism. But the inverse can easily be assembled, so $U$ is an automorphism.

Which of these are to be expected from a simple investigation of the order preserving automorphisms of $G$ ? (See 5.11, 4.32). Such an automorphism (4.31) must send the pair of integers $(0,1)$ onto itself, because this is the least strictly positive element in the ordering 5.11. It appears that we can have precisely ( $k$ any integer)

$$
a(m, n)=(m, n+k m) .
$$

A character $\alpha$ of $G$ sends $f$ into some $z, g$ into some $w,|z|=|w|=1$. There results an automorphism $U$ which by 4.32 makes

$$
U(f)=z f g^{k},
$$

$$
U(g)=w g \text {. }
$$

5.6. TheOREM. The preceding is the most general automorphism of $A_{0}$ obtainable from an automorphism (4.31) of $G$ and a subsequent rotation of $\Gamma$ (i.e., type 4.32-4.35).

Even, therefore, if a general automorphism of $A_{0}$ is required to send the center of $\Delta$ on itself (which requires $b_{0}=0$ in 5.41 ) and to send (as 4.5 stipulates) $\Omega$ on itself (as 5.41 requires, anyway), still

5.7. for this nonarchimedean case, the logical extension of 4.5 does not hold, because $F(g)$ in 5.41 might be taken as $e^{i\left(\theta+o^{-1}\right)}$, for example.

The same example $f F(g)$ serves (of course) also to show that

5.8. in this nonarchimedean case, the condition of 3.4 is not necessary for $\phi$ to be of the form $c \dot{x}$.

\section{BIBLIOGRAPHY}

I. Richard Arens and A. P. Calder6n, Analytic functions of several Banach algebra elements, Ann. of Math. vol. 62 (1955) pp. 204-216.

II. Richard Arens and I. M. Singer, Generalized analytic functions, Trans. Amer. Math. Soc. vol. 81 (1956) pp. 379-393.

III. A. S. Besicovitch, Almost periodic functions, Cambridge, 1932.

UNIVERSITY OF CALIFoRnia

Los ANgeles, Calif.

INGTITUTE FOR ADVANCEd STUdy, Princeton, N.J. 